\title{
Realistic effective interactions for halo nuclei
}

\author{
T.T.S. Kuo ${ }^{1}$, H. Müther ${ }^{2}$, and K. Amir-Azimi-Nili² \\ ${ }^{1}$ Department of Physics, SUNY-Stony Brook \\ Stony Brook, New York 11794 USA \\ 2 Institut für Theoretische Physik, Universität Tübingen \\ D-72076 Tübingen, Germany
}

(October 21, 2019)

\begin{abstract}
We study halo nuclei using a two-frequency shell-model approach employing wave functions of two different oscillator constants $\hbar \omega_{\text {in }}$ and $\hbar \omega_{\text {out }}$, the former for the inner orbits and the latter for the halo (outer) orbits. An initial application has been made for the halo nuclei ${ }^{6} \mathrm{He}$ and ${ }^{6} \mathrm{Li}$, with $0 s_{1 / 2}$ taken as the inner and $\left(0 p_{3 / 2}, 0 p_{1 / 2}\right)$ as the halo orbits. Starting from the Paris NN interaction, we have derived a G-matrix folded-diagram effective interaction for this two-frequency model space, using an essentially exact treatment for the Pauli exclusion operator for the G-matrix. While keeping $\hbar \omega_{\text {in }}$ fixed, we have performed calculations with different choices for $\hbar \omega_{\text {out }}$, treating it as a variation parameter. For $\hbar \omega_{i n}=19.7 \mathrm{MeV}$ and $\hbar \omega_{\text {out }}=8.2 \mathrm{MeV}$, our calculated valence energies for ${ }^{6} \mathrm{He}$ and ${ }^{6} \mathrm{Li}$ are -2.77 and $-3.55 \mathrm{Mev}$, respectively, both in good agreement with experiments. The importance of certain three-body-force diagrams is discussed.
\end{abstract}




\section{INTRODUCTION}

Since several years ago, radioactive-beam nuclear physics has been rapidly developing and has attracted the attention of many nuclear physicists [1 8. With radioactive-beam accelerators, one is now able to measure the properties of a large number of drip-line nuclei, which are far from the nuclear stability valley. These nuclei are generally barely bound (or unbound) and their structure is typically that of a tightly bound inner core with a few outer nucleons which are loosely attached to the core. For example, the halo nucleus ${ }^{6} \mathrm{He}$ is presumably made of a ${ }^{4} \mathrm{He}$ core with a pair of loosely-attached outer nucleons. The outer nucleons are spatially extended and have a large rms radius, forming a "halo" cloud rimming the nucleus as illustrated in Fig. 1. Such nuclei are usually called "halo nuclei". Vaagan et al. [1] have suggested a more distinctive name for them, namely "Borromean nuclei", as a number of halo nuclei, such as ${ }^{11} \mathrm{Li}$, are believed to form a special type of bound three-cluster system.

Studies of halo nuclei, or nuclei far off stability, are of interest in many ways. In astrophysics, the location of the r-process (rapid proton and/or neutron absorption process) path plays a crucial role for nucleosynthesis. The r-process consists of a chain of nuclear reactions, involving a number of intermediate nuclei which are far off stability. To locate the path of the r-process accurately, it is necessary to explore, as much as we can, the various properties of such nuclei. With radioactive beam facilities, the cross section for certain such nuclear reactions important to nucleosynthesis can now be directly measured. For example, the cross section for ${ }^{8} \operatorname{Li}(\alpha, n)^{11} \mathrm{~B}$ has been measured at the radioactive beam facility of RIKEN [9].

Halo nuclei may play also an important role for the synthesis of super-heavy elements, which has been pursued by many physicists. The use of halo nuclei, which are already far off stability, as projectiles may provide a new and possibly more efficient avenue for producing super-heavy elements. Also from a theoretical viewpoint, the study of halo nuclei is of high interest. The empirical nuclear mass formula is, so far, based on a fit to the observed properties of stable nuclei. We need information about halo nuclei to generalize the mass formula to regions far off nuclear stability.

It seems to be still rather uncertain about what would be an appropriate and convenient theoretical framework for the description of halo nuclei. As will be discussed in Section 2, the ordinary nuclear shell model may not be suitable for halo nuclei. We would like to suggest a two-frequency shell model, using single particle (sp) wave functions of two different oscillator constants $\hbar \omega_{\text {in }}$ and $\hbar \omega_{\text {out }}$, the former for the inner orbits while the latter for the halo (outer) orbits.

It is our great pleasure and honor to dedicate this short article to Professor G.E. Brown, or Gerry as known to all his friends, colleagues, students and tennis partners, on the occasion of his 70th birthday. Gerry has pioneered the G-matrix approach for microscopically deriving the nucleon-nucleon effective interaction $\left(V_{e f f}\right)$ in nuclei. [10 12]

This approach has turned out to be quite successful, for ordinary nuclei. For example, the CalTech group has recently carried out shell-model Monte-Carlo calculations [13] using G-matrix effective interactions, with results in remarkably good agreement with experiments over a wide range of nuclei. In this article, we would like to discuss an extension of the above G-matrix approach to halo nuclei, and report some preliminary results.

In the following, we shall first discuss our two-frequency model space and G-matrix together with some calculational details in Section 2. The importance of certain three-body diagrams to the effective interaction will be addressed. In Section 3, we shall present some preliminary results for halo nuclei ${ }^{6} \mathrm{He}$ and ${ }^{6} \mathrm{Li}$. A summary and discussion will be presented in Section 4.

\section{TWO-FREQUENCY MODEL SPACE AND G-MATRIX}

The rms radius for a nucleon in a harmonic-oscillator shell-model orbit $\phi_{n l}$ is given as

$$
r_{r m s}(n l)=\left(2 n+l+\frac{3}{2}\right)^{1 / 2} b ; \quad \text { with } \quad b \equiv(\hbar / m \omega)^{1 / 2}
$$

To fit the observed nuclear radii, there is an empirical formula 14 for choosing the oscillator parameter $\hbar \omega$, namely

$$
\hbar \omega=45 A^{-1 / 3}-25 A^{-2 / 3}
$$

where $\mathrm{A}$ is the nuclear mass number. Using this formula, the empirical value of $\hbar \omega$ is approximately $17.2 \mathrm{MeV}$ for ${ }^{6} \mathrm{He}$, corresponding to a length parameter $\mathrm{b}=1.55 \mathrm{fm}$.

In ordinary shell model calculations, it is customary to use the above empirical formula to determine the $\hbar \omega$ value used for the shell-model wave functions. For example, we may use $\hbar \omega=14 \mathrm{MeV}(\mathrm{b}=1.72 \mathrm{fm})$ for most of the sd-shell nuclei. The situation for halo nuclei is, however, different. For instance, the empirical rms radius for ${ }^{6} \mathrm{He}$ is about 2.5 
fm [i], which corresponds to a b value much larger than the above value of $1.55 \mathrm{fm}$. For halo nuclei such as ${ }^{6} \mathrm{He}$, we need to have wave functions with larger spatial distribution, compared with the ordinary shell-model wave functions.

Let us also look at the binding energies of some nuclei in the vicinity of ${ }^{6} \mathrm{He}$. The observed values [15] are: 28.29 $\left({ }^{4} \mathrm{He}\right), 27.40\left({ }^{5} \mathrm{He}\right), 26.32\left({ }^{5} \mathrm{Li}\right)$, and $29.26\left({ }^{6} \mathrm{He}\right)$, in $\mathrm{MeV}$. We see that the two-neutron separation energy for ${ }^{6} \mathrm{He}$ is only about $1 \mathrm{MeV}$, and the single-nucleon separation energies for ${ }^{5} \mathrm{He}$ and ${ }^{5} \mathrm{Li}$ are both negative (unbound). It is clearly seen that, comparing to ordinary nuclei, ${ }^{6} \mathrm{He}$ is a very "special" breed. It appears to have a tightly-bound ${ }^{4} \mathrm{He}$ core with a pair of very loosely bound outer nucleons. For this situation, it would be very difficult for the ordinary shell model, where all the wave functions have a common $\hbar \omega$ value, to give an adequate description for the nuclear wave function; to have a good description for the ${ }^{4} \mathrm{He}$ core we need to use shell-model wave functions with a small $\mathrm{b}$ value, $\sim 1.45 \mathrm{fm}$, which is much smaller than the $\mathrm{b}$ value needed for a good description for the outer nucleons. In fact, to avoid this serious difficulty, many physicists (see [1] and references quoted therein) have abandoned the ordinary shell-model approach, and employed instead the method of hyperspherical harmonics [1] for the description of halo nuclei.

We would like to consider a two-frequency shell-model (TFSM) approach for halo nuclei, as described below. In recent years, advance has been made for performing realistic nuclear structure calculations starting from a fundamental nucleon-nucleon (NN) potential, such as the Paris potential [16]. The effects of correlations, which are not explicitly included in the model space, are taken into account by deriving the effective hamiltonian for this model space from the realistic NN interaction. For the hyperspherical harmonics method, there is still the restriction that one has to employ a phenomenological effective interaction, such as a Gaussian interaction. This seems to be a drawback. To our knowledge, it is not yet clear how to incorporate realistic NN interactions into the hyperspherical harmonics method. In contrast, for our TFSM approach it seems to be straightforward to perform microscopic calculations for halo nuclei, starting from realistic NN interactions. Otsuka and his coworkers [7] have proposed a variational shell-model approach for treating halo nuclei, and have carried out extensive calculations using the Skyrme effective interactions VSM and SIII.

Let us use the p-shell nuclei ${ }^{6} \mathrm{He}$ and ${ }^{6} \mathrm{Li}$ to illustrate our approach. It is reasonable to consider the "core" of these nuclei as an ordinary alpha particle. Hence we take in the present work the $0 s_{1 / 2}$ orbit as an inner orbit with oscillator constant $\hbar \omega_{i n}=19.7 \mathrm{MeV}$, corresponding a length parameter $b_{i n}=1.45 \mathrm{fm}$. The outer, or halo, nucleons of these nuclei are spatially extended, and for them we use the halo orbits $0 p_{3 / 2}$ and $0 p_{1 / 2}$ with oscillator constant $\hbar \omega_{\text {out }}$, which is treated as a variation parameter. In this way, our sp model space may be written as

$$
P_{s p}=\left\{\phi^{\text {in }}, d^{\text {in }} ; \phi^{\text {out }}, d^{\text {out }}\right\}
$$

with the total dimension of $P_{s p}$ being $d=\left(d^{\text {in }}+d^{\text {out }}\right)$. The superscripts in and out refer to the inner and outer orbits, respectively. Clearly one needs to have all the sp wave functions $\left\{\phi^{i n}, \phi^{\text {out }}\right\}$ be orthonormal to each other, and one may be concerned as to how can one fulfill this condition, as $\hbar \omega_{\text {in }}$ is in general not equal to $\hbar \omega_{\text {out }}$. For a small model space, the orthogonality condition is easily satisfied because of parity and angular momentum conservation. For a general case, we need to use a common $\hbar \omega$ value for all the orbits with the same $l$ and $j$ values. Thus, for our present ${ }^{6} \mathrm{He}$ and ${ }^{6} \mathrm{Li}$ case, we use $\hbar \omega_{i n}$ not only for the $0 s_{1 / 2}$ orbit but also for all the other $s_{1 / 2}$ orbits, in order to fulfill the above orthonormality condition. (Note that the $n s_{1 / 2}$ orbits, with $n>0$, are needed for the intermediate states in the evaluation of the effective interaction as discussed below.)

For a general model space, the model-space effective interaction $V_{\text {eff }}$ can be derived formally from a folded-diagram method [17]. Usually this derivation is carried out in the following three steps. First, we calculate the model-space Brueckner G-matrix defined by the integral equation [18, 19]

$$
G(\omega)=V+V Q_{2} \frac{1}{\omega-Q_{2} T Q_{2}} Q_{2} G(\omega)
$$

where $\omega$ is an energy variable. $Q_{2}$ is a two-body Pauli exclusion operator, and its treatment is very important as we shall later discuss. $T$ is the two-nucleon kinetic energy. Note that our G-matrix has orthogonalized plane-wave intermediate states. In the second step, the irreducible vertex function $\hat{Q}-b o x$ is calculated from the above G-matrix. Finally the energy-independent effective interaction is given by the folded-diagram series

$$
V_{e f f}=\hat{Q}-\hat{Q}^{\prime} \int \hat{Q}+\hat{Q}^{\prime} \int \hat{Q} \int \hat{Q}-\hat{Q}^{\prime} \int \hat{Q} \int \hat{Q} \int \hat{Q} \cdots
$$

where $\int$ denotes a generalized fold, and $\hat{Q}^{\prime}$ and $\hat{Q}$ represent the $\hat{Q}-$ box 17 .

The above formalism is general, and is applicable to either the situation with a single oscillator frequency as in earlier shell-model calculations, or the present situation with two oscillator frequencies. For the latter, the calculation of the G-matrix is more complicated. The exact solution 18 of the G-matrix of Eq.(4) is 


$$
G=G_{F}+\Delta G
$$

where the "free" G-matrix is

$$
G_{F}(\omega)=V+V \frac{1}{\omega-T} G_{F}(\omega)
$$

and the Pauli correction term $\Delta G$ is given by

$$
\Delta G(\omega)=-G_{F}(\omega) \frac{1}{e} P_{2} \frac{1}{P_{2}\left[1 / e+(1 / e) G_{F}(\omega)(1 / e)\right] P_{2}} P_{2} \frac{1}{e} G_{F}(\omega)
$$

where $e=\omega-T$. The projection operator $P_{2}$ is defined as $\left(1-Q_{2}\right)$.

The basic ingredient for calculating the above G-matrix is the matrix elements of $G_{F}$, the free G-matrix, within the $P_{2}$ space. This space is composed of, however, wave functions of two frequencies, $\hbar \omega_{\text {in }}$ and $\hbar \omega_{\text {out }}$. This poses a technical difficulty because transformations from two-particle states in the c.m. coordinates to those in the laboratory coordinates are not as easy to perform as in the case of one oscillator frequency. We have adopted an expansion procedure to surmount this difficulty, namely expanding the oscillator wave functions with $\hbar \omega_{\text {in }}$ in terms of those with $\hbar \omega_{\text {out }}$, or vice versa. When $\hbar \omega_{\text {in }}$ and $\hbar \omega_{\text {out }}$ are close to each other, this procedure is relatively easy to carry out. But when they are significantly different, the two-frequency G-matrix is considerably more complicated to calculate than the one-frequency one.

We write the projection operator $Q_{2}$ as

$$
Q_{2}=\sum_{\text {all } a b} Q(a b)|a b\rangle\langle a b|,
$$

and define $\mathrm{Q}(\mathrm{ab})$ as

$$
Q(a b)= \begin{cases}0, & \text { if } b \leq n_{1}, a \leq n_{3} \\ 0, & \text { if } b \leq n_{2}, a \leq n_{2} \\ 0, & \text { if } b \leq n_{3}, a \leq n_{1} \\ 1, & \text { otherwise }\end{cases}
$$

As shown in Fig. 2a, the boundary of $\mathrm{Q}(\mathrm{ab})$ is specified by the orbital numbers $\left(n_{1}, n_{2}, n_{3}\right)$. We denote the shell model orbits by numerals, starting from the bottom of the oscillator well, 1 for orbit $0 s_{1 / 2}, 2$ for $0 p_{3 / 2}, \ldots 7$ for $0 f_{7 / 2}$ and so on. $n_{1}$ denotes the highest orbit of the closed core (Fermi sea). $n_{2}$ denotes the highest orbit of the chosen model space. We consider here ${ }^{6} \mathrm{He}$ and ${ }^{6} \mathrm{Li}$ with ${ }^{4} \mathrm{He}$ treated as a closed core, thus we have $n_{1}=1$. Suppose we use a model space including all the 6 orbits in the $\mathrm{s}, \mathrm{p}$ and sd shells. Then for this case $n_{2}=6$. In principle one should take $n_{3}=\infty[18]$. In practice, this is not feasible, and one can only use a large $n_{3}$ determined by an empirical procedure. Namely, we perform calculations with increasing values for $n_{3}$ until numerical results become stable. As illustrated in Table 1, we see that there is a convergence behavior and for our present calculation a choice of $n_{3}=21$ appears to be adequate. (This $n_{3}$ value will be used in our present work.)

There is an important point about whether we use a large $n_{3}$ or not. As illustrated in the diagram of Fig. 3 (upper part), we used railed lines to denote nucleons outside the model space while for those inside we use bare lines. This diagram has one line outside the model space, and hence is a legitimate G-matrix diagram, even if the state $b$ refers to a single-particle state below the Fermi energy (i.e. $b \leq n_{1}$ ). We are, however, excluding this diagram from the G-matrix when we use a large $n_{3}$. How can we do this?

There are 3-body components in the effective interaction. Consider the example of the diagram of Fig. 3a (lower part), which represents a 3-body-force (irreducible) diagram, where $j$ denotes, for example, a particle in orbit $0 s_{1 / 2}$ and $a$ represents an orbit outside the model space. (We now draw diagrams with respect to the bare vacuum.) Note that the exchange diagram of (3a) is just diagram (3b) with a minus sign. Diagram (3b) is, however, equivalent to the diagram of Fig. 3 (upper part). Hence the diagrams of the type shown in Fig. 3 (upper part) are exactly canceled by the corresponding 3-body-force diagrams of the type shown in Fig. 3 (lower part). In other words, such 3-body-force diagrams can be incorporated into the G-matrix and in so doing they cancel the G-matrix diagrams of the type shown by Fig. 3 (upper part). Consequently we should use a large $n_{3}$.

It is common to employ a simpler Pauli operator [20] (and references quoted therein), defined by " $Q(a b)=$ 1, if $\left(\rho_{a}+\rho_{b}\right) \leq N_{0} ;=0$, otherwise", with $\rho_{a} \equiv 2 n_{a}+l_{a}$ and similarly for $\rho_{b}$. Such a Pauli operator has the shape shown in Fig. (2b). Here $N_{0}$ is typically a small number, such as 2 for the p-shell nuclei. A main advantage of this choice is its convenience for numerical calculation. However, this choice for $\mathrm{Q}$ corresponds to a small $n_{3}$, and hence the 3-body-force diagrams as shown by Fig. 3 (lower part) are not yet taken into account by the small- $n_{3}$ G-matrix; they have to be calculated separately. In contrast, we use a large $n_{3}$ in our present work, and hence the effect of such 3-body-force diagrams is already absorbed in our G-matrix. Using a small- $n_{3}$ G-matrix alone may over-estimate the nuclear binding energy, as the effect of such 3-body-force diagrams is usually repulsive. 


\section{APPLICATION TO ${ }^{6} \mathrm{HE}$ AND ${ }^{6} \mathrm{LI}$}

Experimental data about ${ }^{6} \mathrm{He}$ and ${ }^{6} \mathrm{Li}$ are rather scarce. The valence energies of these nuclei, defined as

$$
E_{\text {val }}\left({ }^{6} \mathrm{He}\right)=-\left[B E\left({ }^{6} \mathrm{He}\right)+B E\left({ }^{4} \mathrm{He}\right)-2 \times B E\left({ }^{5} \mathrm{He}\right)\right]
$$

and

$$
E_{\text {val }}\left({ }^{6} \mathrm{Li}\right)=-\left[B E\left({ }^{6} \mathrm{Li}\right)+B E\left({ }^{4} \mathrm{He}\right)-B E\left({ }^{5} \mathrm{He}\right)-B E\left({ }^{5} \mathrm{Li}\right)\right],
$$

are nevertheless well known. From the empirical binding energies (BE) [15], the valence energy for ${ }^{6} \mathrm{He}$ is found as $-2.77 \mathrm{MeV}$ and that for ${ }^{6} \mathrm{Li}$ is $-3.83 \mathrm{MeV}$.

We want to calculate these quantities using a TFSM approach. For the inner orbit $0 s_{1 / 2}$ we use $b_{i n}=1.45 \mathrm{fm}$. For the outer orbits $0 p_{3 / 2}$ and $0 p_{1 / 2}$ we use a sequence of $b_{\text {out }}$ values, ranging from 1.45 to $2.25 \mathrm{fm}$. The G-matrix is first calculated using a Pauli operator specified by $\left(n_{1}, n_{2}, n_{3}\right)=(1,6,21)$, starting from the Paris potential. Then, following closely the $\hat{Q}-$ box folded-diagram procedure of Shurpin et al. [21], we derive the matrix elements of the effective interaction $V_{e f f}$ for the p-shell. (In the $\hat{Q}-b o x$ we have included diagrams first- and second-order in G. This includes the diagram of second order in $G$ with an intermediate state of two nucleons in $1 s 0 d$ shell. To avoid overcounting we have to use $n_{2}=6$ in the Pauli operator for the G-matrix equation.) Our results for the matrix elements of $V_{\text {eff }}$ are listed in Table 2. As expected, these matrix elements are seen to become generally weaker as $b_{\text {out }}$ increases, a reflection that the mean distance between the interacting valence nucleons is larger. The empirical (6-16) matrix elements of Cohen and Kurath 24] are also listed for comparison. For some cases, the agreement between our calcualated matrix elements and their values becomes improved when we use a $b_{\text {out }}$ value larger than $1.45 \mathrm{fm}$.

To obtain the valence energies, we just diagonalize the two-particle matrix $\left(H_{0}+V_{\text {eff }}\right)$ within the p-shell, using the matrix elements of Table 2. $H_{0}$ is the sp Hamiltonian. For the valence-energy calculation, the single-particle energy for $p_{3 / 2}$ is defined as zero. But that for $p_{1 / 2}$ is uncertain, both experimentally and theoretically. For an initial calculation, we have fixed it as $10 \mathrm{MeV}$. (Using an empirical formula given in Bohr and Mottelson's book [22], the $p_{1 / 2}-p_{1 / 3}$ spin-orbit splitting is estimated to be $\sim 10.2 \mathrm{MeV}$.) The valence energy for ${ }^{6} \mathrm{He}$ is then just the lowest eigenvalue of the $\mathrm{T}=1, \mathrm{~J}=0$ secular matrix, and that of the $\mathrm{T}=0, \mathrm{~J}=1$ matrix gives the valence energy for ${ }^{6} \mathrm{Li}$. Our results for these energies are displayed in the lower part of Fig. 4, calculated as a function of $b_{\text {out }}$. At $b_{\text {out }} \simeq 1.45$ $\mathrm{fm}$, the calculated values are too large. At $b_{\text {out }}=2.25 \mathrm{fm}$, our results are -2.77 and $-3.55 \mathrm{MeV}$ for ${ }^{6} \mathrm{He}$ and ${ }^{6} \mathrm{Li}$, respectively, both in reasonable agreement with the corresponding empirical values -2.76 and $-3.83 \mathrm{MeV}$.

We have also calculated the single-particle separation energy of ${ }^{5} \mathrm{He}$, defined as $E_{s p}=B E\left({ }^{5} \mathrm{He}\right)-B E\left({ }^{4} \mathrm{He}\right)$. Our calculation was done using a one-body $\hat{Q}$ - box consisting of the three 1-body diagrams (first- and second-order in G) of Ref. 21] and then summing up the folded-diagram series consisting of the one-body $\hat{Q}-$ box. These results are presented in the upper part of Fig. 4. There is an improvement of our results as $b_{\text {out }}$ increases. However, our calculated separation energy is still too large at $b_{\text {out }} \simeq 2.25 \mathrm{fm}$. It is of interest to note that there seems to be a saturation point for $E_{s p}$ at $b_{\text {out }} \simeq 2.25 \mathrm{fm}$. We recall that at this $b_{\text {out }}$ our results for $E_{\text {val }}$ are also in good agreement with experiments. Assuming a pure two-frequency $s^{4} p^{2}$ wave function with $b_{\text {in }}=1.45$ and $b_{\text {out }}=2.25 \mathrm{fm}$, the $\mathrm{rms}$ radius for ${ }^{6} \mathrm{He}$ is obtained, using Eq.(1), as $2.37 \mathrm{fm}$, which is also in reasonable agreement with the empirical value of $\sim 2.5 \mathrm{fm}$ [1].

Encouraged by the above preliminary results, we have begun to tackle a more difficult problem, to calculate the total binding energies of ${ }^{4} \mathrm{He},{ }^{5} \mathrm{He}$ and ${ }^{6} \mathrm{He}$ using our present two-frequency approach. Here we employ a two-shell ( $\mathrm{s}$ and $\mathrm{p}$ ) model space, treating all nucleons of the nucleus as active. For s-shell we use fixed length parameter $b_{i n}=$ $1.45 \mathrm{fm}$. For p-shell we use a varied $b_{\text {out }}$. The components of a spurious c.m. motion, which are contained in such a model space including valence orbits of 2 major shells are removed using the technique of Gloeckner and Lawson [23]. Our preliminary results indicate that for ${ }^{4} \mathrm{He}$ an energy minimum seems to be at $b_{\text {out }} \simeq 1.45 \mathrm{fm}$, while for ${ }^{6} \mathrm{He}$ the minimum seems to be located at $b_{\text {out }} \simeq 2.25 \mathrm{fm}$. Our calculated total binding energies have turned out to be too small, for both nuclei, compared with experiments. This is perhaps a common problem of nuclear structure calculations with no-core employing realistic NN interactions. Further studies are being pursued, and we hope to report our results before long.

\section{SUMMARY AND DISCUSSION}

Halo nuclei have a special nuclear structure; they have typically a tightly bound core with a few halo nucleons loosely attached to the core. One would encounter a lot of difficulties, in describing such nuclei using the conventional 
shell model where the basis functions all have the same $\hbar \omega$ value. For example, the wave functions with $b_{i n}=1.45$ $\mathrm{fm}$ would give a good description for the alpha-particle core of ${ }^{6} \mathrm{He}$, but they are spatially much too compressed to describe the halo neutrons of ${ }^{6} \mathrm{He}$. If one insists to use the compressed wave functions also for the halo nucleons, then one needs to use a very large active space (such as a space with s, p, sd and pf shells for the case of ${ }^{6} \mathrm{He}$ ). This would be very tedious as well as not economical. A much simpler solution is to allow for a two-frequency model space, where one uses $b_{\text {in }}$ for the core and $b_{\text {out }}$ for the halo part of the nucleus as described in the present work. We have obtained some preliminary results using this approach. Our results seem to indicate that this two-frequency method is a feasible and promising approach for halo nuclei.

What effective interactions should one use in a two-frequency shell-model calculation? It would be very difficult to determine them by an empirical best-fit procedure, which has been successfully used for one-frequency shell-model calculations [25]. This is because now the length parameter $b_{\text {out }}$ is a variable and the matrix elements of the effective interaction are dependent on it. A basic and rather "ambitious" solution to this problem would be to derive the effective interaction directly from a fundamental NN interaction. We have carried out some preliminary studies in this direction. We first calculate the two-frequency G-matrix, with an accurate treatment for its two-frequency Pauli exclusion operator. Then the effective interaction is obtained via a standard folded-diagram method. Our calculated valence energies for ${ }^{6} \mathrm{He}$ and ${ }^{6} \mathrm{Li}$ are in satisfactory agreement with experiments. As indicated by our preliminary results, ${ }^{4} \mathrm{He}$ seems to favor a one-frequency $\left(b_{\text {in }}=b_{\text {out }}=1.45 \mathrm{fm}\right)$ shell model, while a two-frequency $\left(b_{\text {in }}=1.45, b_{\text {out }}=2.25\right.$ fm) one is perhaps needed for ${ }^{6} \mathrm{He},{ }^{6} \mathrm{Li}$ and ${ }^{5} \mathrm{He}$.

\section{Acknowledgements}

Part of this work was performed when TTSK was a summer visitor at Tübingen. He would like to thank Professors A. Faessler and H. Müther for their warm hospitality during his stay at Tübingen. This work is supported in part by "Graduiertenkolleg Struktur und Wechselwirkung von Hadronen und Kernen" (DFG Mu 705/3) and by the USDOE Grant DE-FG02-88ER40388.

[1] M.V. Zhukov, B. Danilin, D.V. Fedorov, J.M. Bang, I.J. Thompson and J.S. Vaagan, Phys. Rep. 231, 151 (1993)

[2] A.C. Mueller and B.M. Sherril, Ann. Rev. Nucl. Part. Sci. 43, 529 (1993)

[3] R. N. Boyd, Int. J. Mod. Phys. 3 249, (1994)

[4] G.F. Bertsch, B.A. Brown and H. Sagawa, Phys. Rev. C39, 1154 (1989)

[5] Z. Ren, M. Mittig, B. Chen, Z. Ma, G. Auger and G. Xu, Phys. Rev. C52, R1764 (1995)

[6] H. Nakada and T. Otsuka, Phys. Rev. C49, 886 (1994)

[7] T. Otsuka and N. Fukunishi, Phys. Rep. 264, 297 (1996)

[8] W.E. Ormand, Phys. Rev. C53, 214 (1996)

[9] R. N. Boyd et al., Phys. Rev. Lett. 68,1283 (1992)

[10] G.E. Brown, Unified Theory of Nuclear Models and Forces, North Holland Publ. Co. (1967)

[11] G.E. Brown and T.T.S. Kuo, Nucl. Phys. A92, 481 (1967)

[12] T.T.S. Kuo and G.E. Brown, Nucl. Phys. 85, 40 (1966)

[13] K. Langanke, D.J. Dean, P.B. Radha, Y. Alhassid, and S.E. Koonin, Phys. Rev. C 52, 718 (1995); D.J. Dean, S.E. Koonin, T.T.S. Kuo, K. Langanke, and P.B. Radha, Phys. Lett. B367, 17 (1996)

[14] G.F. Bertsch, The Practioner's Shell Model, North Holland (1972)

[15] J.K. Tuli (editor), Nuclear Wallet Cards, National Nuclear Data Center, Brookhaven National Laboratory (1995)

[16] M. Lacombe et al., Phys. Rev. C21 (1980) 861

[17] T.T.S. Kuo and E. Osnes, Lecture Notes in Physics Vol.364 (Springer-Verlag 1990) p.1

[18] E.M. Krenciglowa, C.L. Kung, T.T.S. Kuo, and E. Osnes, Ann. Phys. (N.Y.)101, 154 (1976)

[19] H. Müther and P. Sauer, in Computational Nuclear Physics 2, ed. by K. Langanke, J.A. Maruhn and S. Koonin, SpringerVerlag 1992, p.30

[20] D.C. Zheng, B.R. Barrett, J.P. Vary, W.C. Haxton and H.S. Song, Phys. Rev. C52, 2488 (1995)

[21] J. Shurpin, T.T.S. Kuo, and D. Strottman, Nucl. Phys. A408, 310(1983)

[22] A. Bohr and B. Mottelson, Nuclear Structure, Vol. 1, W.A. Benjamin (1969)

[23] D.H. Gloeckner and R.D. Lawson, Phys. Lett. 53 B, 313 (1974)

[24] S. Cohen and D. Kurath, Nucl. Phys. 73, 1 (1965)

[25] B.A. Brown and B.H. Wildenthal, Ann. Rev. Nucl. Part. Sci. 38, 29 (1988) 


\begin{tabular}{|c|c|c|c|c|}
\hline & & & $\bar{\omega}[\mathrm{MeV}]$ & \\
\hline$a b c d T J$ & $n_{3}$ & -5 & -10 & -20 \\
\hline$\overline{111101}$ & 3 & -16.048 & -15.424 & -14.453 \\
\hline 1111101 & 6 & -15.295 & -14.706 & -13.842 \\
\hline 1111101 & 15 & -15.238 & -14.637 & -13.767 \\
\hline 1111101 & 21 & -15.238 & -14.637 & -13.767 \\
\hline 232302 & 3 & -6.138 & -5.691 & -5.132 \\
\hline 232302 & 6 & -5.635 & -5.339 & -4.921 \\
\hline 232302 & 15 & -5.563 & -5.277 & -4.877 \\
\hline 232302 & 21 & -5.563 & -5.277 & -4.877 \\
\hline 111110 & 3 & -9.456 & -9.360 & -9.213 \\
\hline 1111110 & 6 & -9.151 & -9.093 & -8.995 \\
\hline 111110 & 15 & -9.140 & -9.083 & -8.986 \\
\hline 1111110 & 21 & -9.140 & -9.083 & -8.986 \\
\hline
\end{tabular}

Table 1. Dependence of the two-frequency G-matrix on the choice of $n_{3}$. Listed are the matrix element $\langle a b T J|G(\omega)| c d T J\rangle$, in $\mathrm{MeV}$, calculated with the Paris potential. The orbits $1,2,3$ represent, respectively, $0 s_{1 / 2}, 0 p_{3 / 2}, 0 p_{1 / 2}$. We have used $b_{i n}=1.45$ and $b_{\text {out }}=2.0 \mathrm{fm}$ for the length parameters, and $n_{1}=1$ and $n_{2}=3$ for the exclusion operator. 


\begin{tabular}{|c|c|c|c|c|c|}
\hline & & & $\overline{b_{\text {out }}[\mathrm{fm}]}$ & & 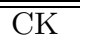 \\
\hline$a b c d T J$ & 1.45 & 1.75 & 2.00 & 2.25 & \\
\hline 222201 & -1.76 & -2.15 & -2.28 & -2.24 & -3.14 \\
\hline 222301 & 5.64 & 4.69 & 3.89 & 3.18 & 4.02 \\
\hline 223301 & 2.55 & 2.24 & 2.13 & 2.03 & 1.09 \\
\hline 2322201 & 5.81 & 4.64 & 3.78 & 3.05 & 4.02 \\
\hline 232301 & -7.63 & -6.75 & -5.96 & -5.15 & -6.54 \\
\hline 233301 & 3.12 & 1.72 & 0.83 & 0.20 & 1.39 \\
\hline 332201 & 2.72 & 2.20 & 2.01 & 1.87 & 1.09 \\
\hline 332301 & 3.11 & 1.82 & 1.08 & 0.56 & 1.39 \\
\hline 333301 & -3.13 & -2.80 & -2.61 & -2.37 & -4.26 \\
\hline 222210 & -3.43 & -3.28 & -2.92 & -2.51 & -2.74 \\
\hline 223310 & -5.21 & -3.88 & -3.02 & -2.38 & -5.32 \\
\hline 332210 & -5.41 & -3.86 & -2.99 & -2.36 & -5.32 \\
\hline 333310 & 0.44 & -0.45 & -0.74 & -0.81 & 0.34 \\
\hline
\end{tabular}

Table 2. Some p-shell effective interactions calculated with various $b_{\text {out }}$ values. Listed are the matrix element $\left\langle a b T J\left|V_{\text {eff }}\right| c d T J\right\rangle$, calculated from the Paris potential. The orbits 2,3 represent, respectively, $0 p_{3 / 2}, 0 p_{1 / 2}$. We have used $b_{i n}=1.45 \mathrm{fm}$ and bare G-matrix calculated with $\left(n_{1}, n_{2}, n_{3}\right)=(1,6,21)$. The empirical (6-16) matrix elements of Cohen and Kurath 24] are listed under column CK. 


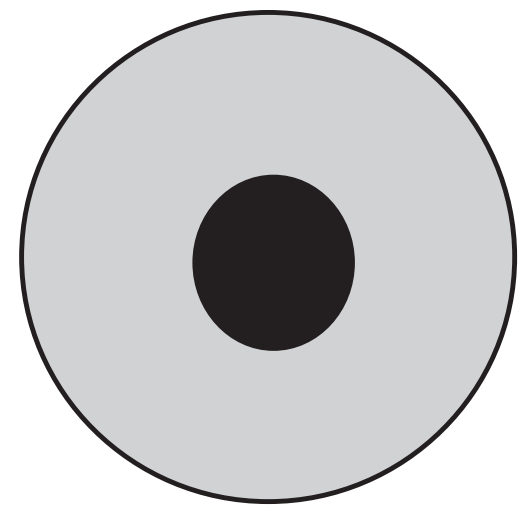

FIG. 1. A halo nucleus.

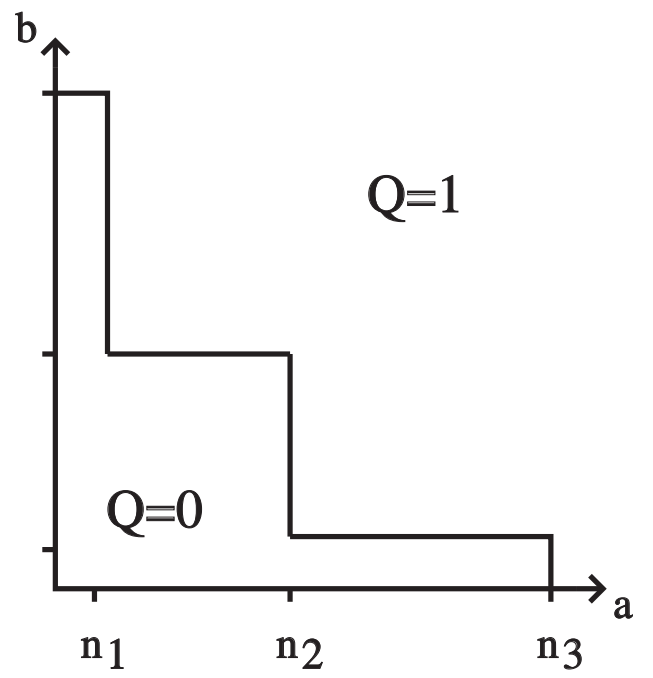

(a)

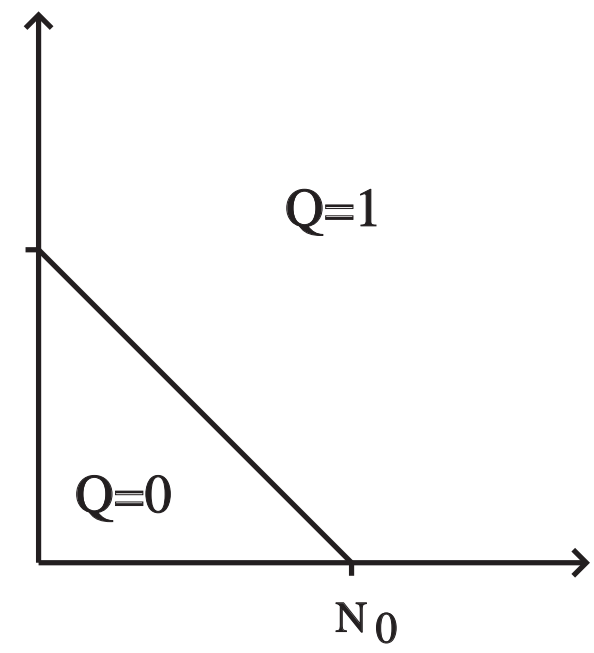

(b)

FIG. 2. Pauli exclusion operator $Q_{2}$. 


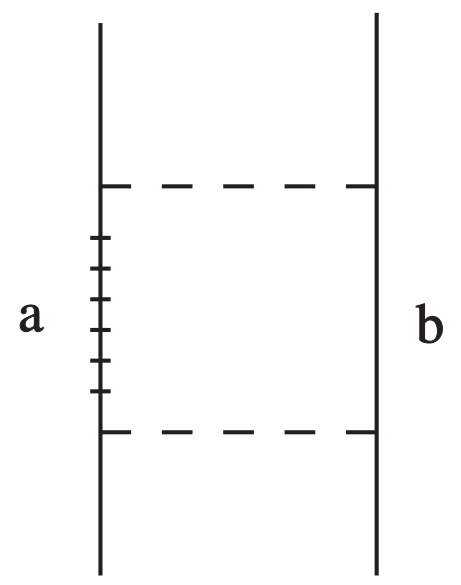

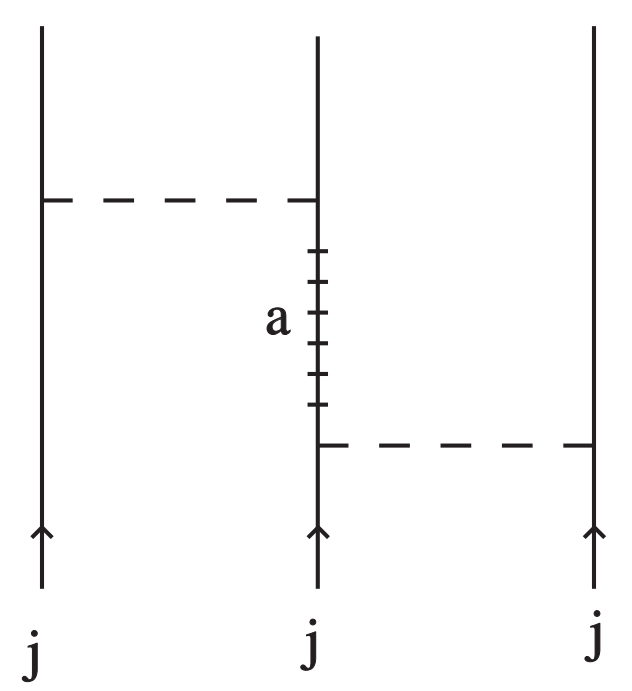

(a)

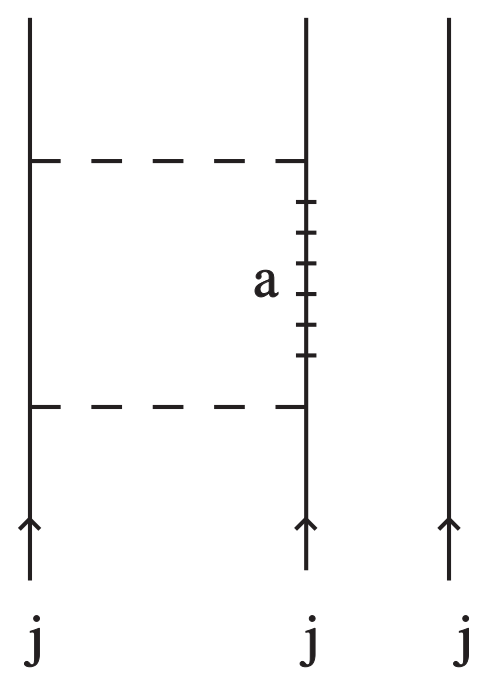

(b)

FIG. 3. A second-order diagram belonging to the G-matrix (upper part) and Three-body-force diagrams (lower part). 

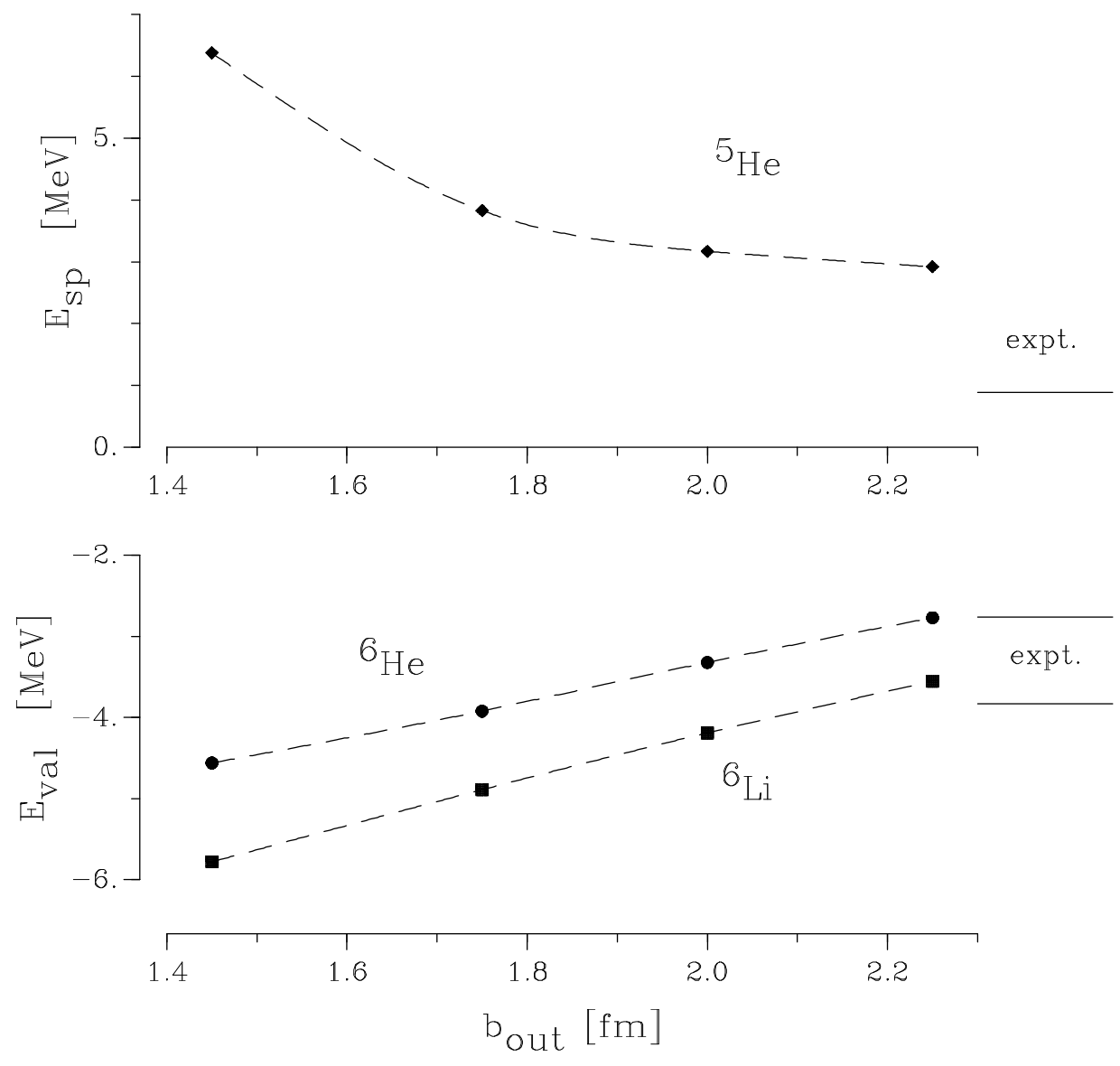

FIG. 4. The lower part of this figure shows the valence energy $E_{v a l}$ for ${ }^{6} \mathrm{He}$ and ${ }^{6} \mathrm{Li}$ as a function of $b_{\text {out }}$, while the upper part displays the single-particle separation energy $E_{s p}$ for ${ }^{5} \mathrm{He}$ as a function of $b_{\text {out }}$. 